\title{
Robotic-assisted transanal repair of rectourethral fistula
}

Rectourethral fistulas represent an uncommon but severe complication of pelvic surgery, especially after radical prostatectomy, radiation therapy, and accidental intraoperative rectal injury. These fistulas usually present large $(>2 \mathrm{~cm})$, complex, fibrotic tissue, and they are difficult to treat with conservative treatment [1]. Here, we present a new operative technique to successfully treat a patient with rectourethral fistula ( $\mathbf{V i}$ deo 1).

This case was a 52-year-old man who was diagnosed with prostate cancer in September 2015. For curative intent, he underwent laparoscopic radical prostatectomy with bilateral pelvic lymph node dissection in December 2015. In January 2017, he complained of anal urinary leakage during micturition. Further computed tomography cystogram was scheduled, and the image indicated a fistula between the rectum and urethra (॰ Fig. 1).

For the first step, we arranged cystoscopy to orientate the urethral opening of the fistula. Then a guidewire (Glidewire; Terumo Medical, Tokyo, Japan) was positioned into the fistula opening (> Fig. 2) and out through the rectal side of the fistula. A communication via the guidewire was created, and we could easily trace the fistula route. Subsequently, the da Vinci Surgical System (Intuitive Surgical Inc., Sunnyvale, California, USA) was docked over the anus with a disposable single-port platform. Tracing the rectal side of the fistula along the guidewire, the fistulectomy with "core-out" technique was performed ( $\mathbf{F i g . 3} \mathbf{a}$ ), and the fistula tract was carefully dissected from the rectal opening to the urethral opening ( $\triangleright$ Fig.3 b). We then vertically sutured the urethral wound, sealed the tunnel with fibrin sealant (Tisseel; Baxter Healthcare Corp., Deerfield, Illinois, USA) (> Fig. $3 \mathrm{c})$, and horizontally closed the rectal wound ( $\triangleright$ Fig. $3 d$ ). Finally, a thin fibrin

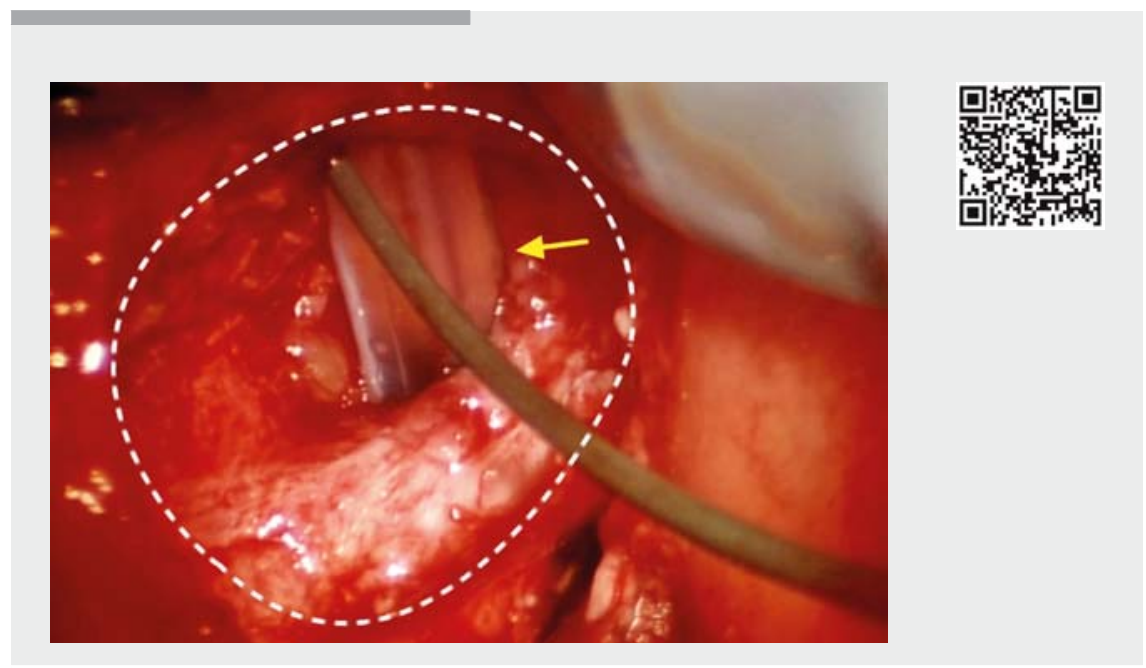

$\checkmark$ Video 1 The surgical technique of robotic-assisted transanal repair of rectourethral fistula. The silicone Foley catheter (yellow arrow) indicates that the fistula dissection between the rectum and urethra (white dashed line) is complete.

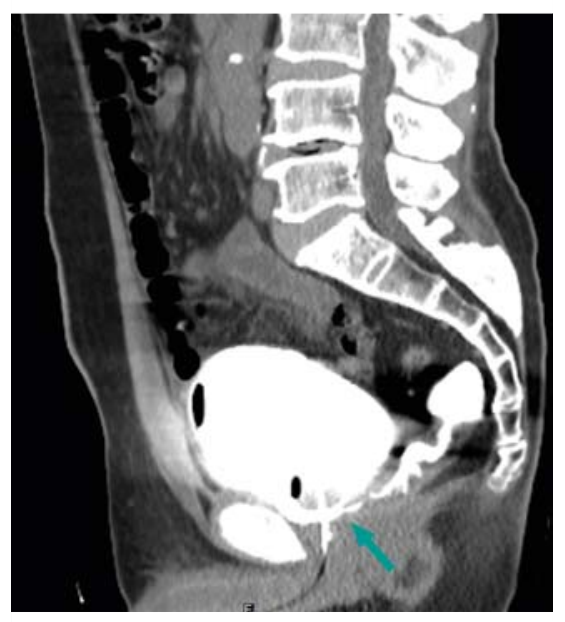

Fig. 1 Computed tomography cystogram showing rectourethral fistula formation (arrow).

sealant was applied to the sutured wound on the rectal side. No evidence of recurrent rectourethral fistula was recorded at 3 and 12 months' follow-up.

Robotic-assisted transanal repair of rectourethral fistula is a feasible surgical technique and an alternative to the tradi-

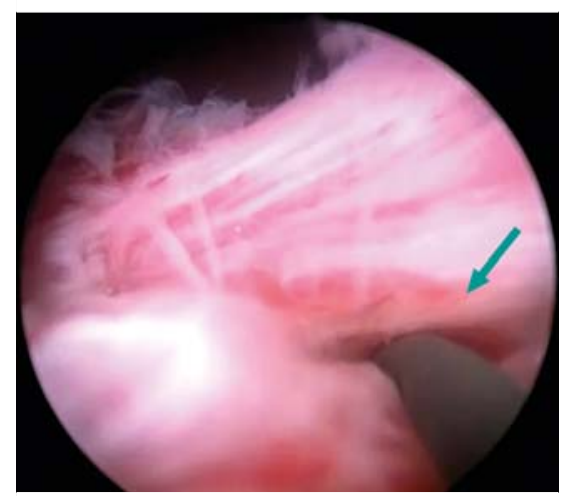

- Fig. 2 Cystoscopic view showing the urethral opening of the fistula (arrow) with a guidewire. tional perineal approach or the York Mason procedure. The biggest advantage of this surgical technique is that it does not require the flap repair or fecal diversion with a temporary colostomy.

Endoscopy_UCTN_Code_TTT_1AQ_2AG 

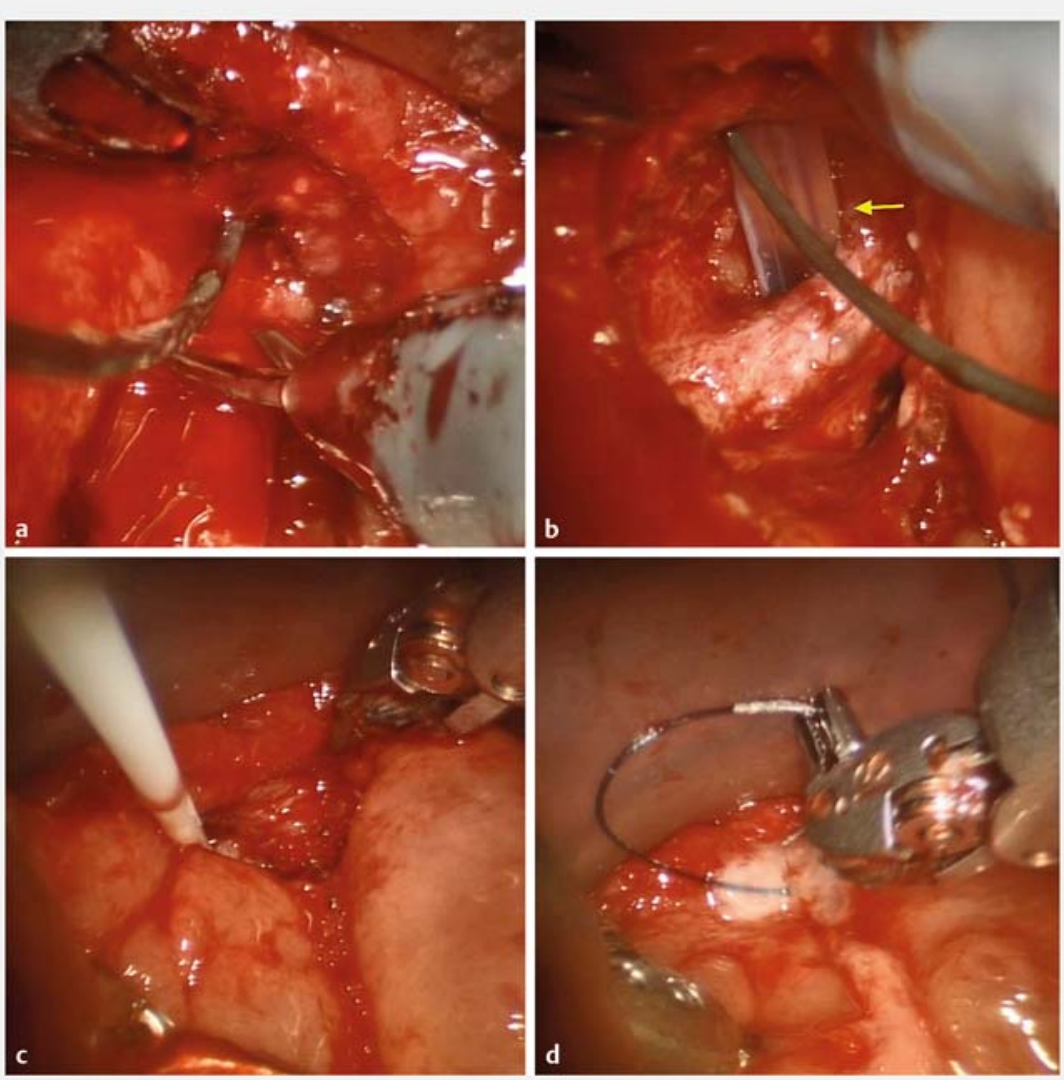

Fig. 3 The views of the da Vinci Surgical System (Intuitive Surgical Inc., Sunnyvale, California, USA). a Fistulectomy with "core-out" technique alongside the guidewire. b Visualization of the silicone Foley catheter (arrow) confirmed the dissection to the urethral side. c Sealing the fistulous tunnel with fibrin sealant. $\mathbf{d}$ Closing the rectal wound after tunnel sealing.

\section{Competing interests}

None

The authors

\section{Shih-I Tseng ${ }^{1,2}$, Ching-Wen Huang ${ }^{3,4}$, Tsung-Yi} Huang ${ }^{1,2}$

1 Department of Urology, School of Medicine, College of Medicine, Kaohsiung Medical University, Kaohsiung, Taiwan

2 Department of Urology, Kaohsiung Medical University Hospital, Kaohsiung Medical University, Kaohsiung, Taiwan
3 Graduate Institute of Medicine, College of Medicine, Kaohsiung Medical University, Kaohsiung, Taiwan

4 Division of Colorectal Surgery, Department of Surgery, Kaohsiung Medical University Hospital, Kaohsiung Medical University, Kaohsiung, Taiwan

\section{Corresponding author}

\section{Tsung-Yi Huang, MD}

Department of Urology, School of Medicine, Kaohsiung Medical University, 100 ShihChuan 1st Road, Kaohsiung 80708, Taiwan Fax: +886-7-3211033

sculptor39@yahoo.com.tw

\section{Reference}

[1] Hechenbleikner EM, Buckley JC, Wick EC. Acquired rectourethral fistulas in adults: a systematic review of surgical repair techniques and outcomes. Dis Colon Rectum 2013; 56: $374-383$

\section{Bibliography}

DOI https://doi.org/10.1055/a-0826-4220

Published online: 12.2.2019

Endoscopy 2019; 51: E96-E97

(c) Georg Thieme Verlag KG

Stuttgart · New York

ISSN 0013-726X

\section{ENDOSCOPY E-VIDEOS \\ https:|/eref.thieme.de/e-videos}

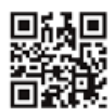

Endoscopy E-Videos is a free access online section, reporting on interesting cases and new techniques in gastroenterological endoscopy. All papers include a high quality video and all contributions are freely accessible online.

This section has its own submission website at https://mc.manuscriptcentral.com/e-videos 